\title{
La objetivación de la noción de sector circular en el trabajo matemático con GeoGebra
}

\author{
Ivonne C. Sánchez S. ${ }^{1,2}$ \\ ivonne.s.1812@gmail.com \\ https://orcid.org/0000-0002-2485-1059 \\ João Cláudio Brandemberg Quaresma ${ }^{1}$ \\ brand@ufpa.br \\ https://orcid.org/0000-0001-8848-3550 \\ Luis Andrés Castillo B., ${ }^{1,2}$ \\ luiscastleb@gmail.com \\ https://orcid.org/0000-0002-5174-9148 \\ ${ }^{1}$ Universidade Federal do Pará (UFPA, Brasil) \\ ${ }^{2}$ Asociación Aprender en Red (Venezuela)
}

Recibido: 31/05/2020 Aceptado: 04/07/2020

\begin{abstract}
Resumen
Este artículo presenta resultados obtenidos de una investigación en el desarrollo de una tesis de maestría, titulada "Aprendizaje geométrico sobre ideales presentes en la simulación de un motor de dos tiempos en GeoGebra: Un estudio de caso". Referida investigación fue desarrollada en el Programa de Posgrado en Educación en Ciencias y Matemáticas de la Universidad Federal de Pará. En este artículo se describen los procesos de objetivación de la noción geométrica del sector circular manifestado por un grupo de estudiantes de la Licenciatura Integrada en Ciencias, Matemáticas y Lenguaje en actividades de Elaboración de Simuladores con GeoGebra (ESG). Específicamente, la objetivación del referido contenido geométrico se evidenció en las actividades de trabajo matemático llevadas a cabo para simular el cigüeñal de un motor de dos tiempos en el GeoGebra. Este estudio tomó como fundamento teórico la Teoría de la Objetivación, para tener una óptica que permitiese evidenciar la objetivación del conocimiento geométrico para describir el grupo de estudiante llegan al encuentro con la noción de sector circular al simular un cigüeñal de un motor de dos tiempos en GeoGebra. Los datos de la investigación provienen de las grabaciones realizadas en las sesiones de trabajo con estudiantes y profesores cuando realizaban las actividades, particularmente en el momento de trabajo matemático. Realizamos un análisis multisemiótico de la información obtenida de las transcripciones hechas de las grabaciones. Los resultados se centran en cómo los estudiantes usan medios semióticos de objetivación para expresar y dar forma a la noción de sector circular. Palabras clave: Procesos de objetivación; Medios semióticos; GeoGebra; Sector Circular.
\end{abstract}

\section{A objetivação da noção do setor circular no trabalho matemático da GeoGebra}

\section{Resumo}

Este artigo apresenta resultados obtidos em uma investigação no desenvolvimento de uma dissertação de mestrado, intitulada "Aprendizado geométrico sobre ideais atuais na simulação 
de um motor a dois tempos no GeoGebra: um estudo de caso". Esta pesquisa foi desenvolvida no Programa de Pós-Graduação em Ensino de Ciências e Matemática da Universidade Federal do Pará. Este artigo descreve os processos de objetivação da noção geométrica do setor circular manifestada por um grupo de estudantes de uma turma da Licenciatura Integrada de Ciências, Matemática e Linguagem em atividades de Elaboração de Simuladores com GeoGebra (ESG). Especificamente, a objetificação do conteúdo geométrico mencionado acima foi evidenciada nas atividades matemáticas de trabalho realizadas para simular o virabrequim de um motor de dois tempos no GeoGebra. Este estudo tomou a Teoria da Objetivação como fundamento teórico, para ter uma ótica que permitisse descrever a objetivação do conhecimento geométrico para descrever o grupo de alunos que eles encontram a noção de setor circular ao simular uma cambota de um motor a dois tempos. GeoGebra. Os dados da pesquisa provêm das gravações realizadas nas sessões de trabalho com alunos e professores quando elas realizavam as atividades, principalmente no momento do trabalho matemático. Foi realizada uma análise multissemiótica das informações obtidas das transcrições feitas a partir das gravações. Os resultados se concentram em como os alunos usam meios semióticos de objetivação para expressar e moldar a noção de setor circular.

Palavras-chave: Processos de objetificação; Meios semióticos; GeoGebra; Setor Circular.

\title{
The objectivation of the notion of circular sector in mathematical work with GeoGebra
}

\begin{abstract}
This article presents results obtained from an investigation in the development of a master's thesis, titled "Geometric learning about present ideals in the simulation of a two-stroke engine in GeoGebra: A case study". This research was carried out in the Graduate Program in Science and Mathematics Education of the Federal University of Pará. This article describes the processes of objectification of the geometric notion of the circular sector manifested by a group of students of the Integrated Degree in Science, Mathematics and Language in activities of Simulator Development with GeoGebra (ESG). Specifically, the objectification of the referred geometric content was evidenced in the mathematical work activities carried out to simulate the crankshaft of a two-stroke engine in GeoGebra. This study took as a theoretical foundation the Theory of the Objectification, to have an optics that would allow the objectification of geometric knowledge to be described to describe the group of students they come across the notion of circular sector when simulating a crankshaft of a two-stroke engine in GeoGebra. The research data comes from the recordings made in the work sessions with students and teachers when they carried out the activities, particularly at the time of mathematical work. We performed a multisemiotic analysis of the information obtained from the transcripts made from the recordings. The results focus on how students use semiotic means of objectification to express and shape the notion of circular sector.
\end{abstract}

Keywords: Objectification processes; Semiotic media; GeoGebra; Circular Sector. 


\section{Introducción}

El aprendizaje matemático es un fenómeno educativo cuya caracterización depende de la perspectiva teórica con la que decide ser analizado. Una perspectiva teórica que ha tenido una gran influencia en la forma de entender el aprendizaje matemático desde finales del siglo XX es el constructivismo. Sin embargo, a lo largo de los años, se han desarrollado nuevas perspectivas de aprendizaje para responder a la necesidad de integrar estos aspectos sociales, culturales e históricos en el aprendizaje de los estudiantes. Una de estas perspectivas es sociocultural, que se caracteriza, por un lado, porque el conocimiento es generado por individuos en el curso de prácticas sociales que son consustanciales en la historia y la cultura.

Un ejemplo de estas perspectivas socioculturales que permitieron mirar la educación matemática con otros lentes, es la teoría histórico-cultural de la enseñanza y el aprendizaje de las matemáticas, denominada Teoría de la Objetivación (TO), desarrollada por Radford (2014, 2020). La TO se basa una concepción social del aprendizaje, en la cual los sujetos involucrados en alguna actividad de enseñanza-aprendizaje, desarrollan capacidades intelectuales mientras aprenden a relacionarse con los otros, es decir, también se promueven capacidades de relaciones humanas. Además, defiende una concepción no mentalista del pensamiento, en la que se puede observar, ya que emerge a través de gestos, movimientos corporales, actividad perceptiva, artefactos y signos utilizados por individuos y no como algo inobservable, que solo sucede en el plano mental.

Desde la perspectiva de la TO, el pensamiento se enmarca en significados culturales que guían la actividad de los individuos y les dan una cierta forma. Una forma que se refleja en la forma de demostrar, argumentar y resolver problemas, ya que tiene significados culturales históricos que la preceden, es decir, esta forma de actividad está sustentada por una superestructura simbólica que se ha denominado medios semióticos. Estos medios ayudan a comprender las concepciones de los objetos matemáticos, su existencia, la relación con el mundo concreto y los patrones sociales de producción de significados culturales (Radford, 2006).

Recientemente, la perspectiva sociocultural ha animado el debate sobre el papel desempeñado por el trabajo humano en el desarrollo de formas histórico-culturales de producir conocimiento matemático, independientemente de las condiciones institucionales que rodean la 
actividad. En este sentido, actividades educativas, formales y no formal, como la Elaboración de Simuladores con GeoGebra (ESG), se consideran una instancia social del encuentro con el saber matemático, el posicionamiento crítico ante ellos (Prieto G., \& Ortiz, 2019) y como un escenario para la promover formas de colaboración humanas en la ESG (Castillo \& Sánchez, 2020; Prieto G., Castillo \& Márquez, 2020).

Aunque la alienación nunca ha sido el objetivo de la ESG, consideramos importante evitarla tanto en la medida de lo posible y, para esto, es necesario llevar a cabo estudios que nos permitan ampliar nuestra comprensión de las implicaciones de la ESG en el aprendizaje geométrico de los estudiantes que participan en la actividad. Asumir una perspectiva sociocultural para esto implica prestar especial atención al papel de la cultura en la producción del conocimiento matemático escolar, es decir, la forma los individuos involucrados en la actividad piensan y actúan juntos con respecto al conocimiento matemático, utilizando una serie de recursos semióticos organizados en la cultura escolar. Una perspectiva nos permitirá ampliar nuestras comprensiones de las implicaciones de la ESG en el aprendizaje geométrico de los estudiantes que participaron.

Por lo anteriormente expuesto, el objetivo de este trabajo es describir la objetivación de la noción de sector circular que ocurre en las actividades de trabajo matemático en la ESG, realizada por un grupo de estudiantes de pregrado junto a sus profesores. Nuestra intención es contribuir con información que sea beneficiosa para los profesores que están realizando la ESG como una oportunidad para promover procesos de enseñanza-aprendizaje de la matemática.

\section{Marco Teórico}

Para conseguir el objetivo de la investigación, nos apoyamos en una perspectiva histórico-cultural del aprendizaje matemático, expresada en la Teoría de la Objetivación (TO) por Luis Radford (2006, 2014, 2020), para interpretar el aprendizaje geométrico producido en las actividades de trabajo matemático llevadas a cabo por estudiantes de pregrado y profesores que resolvieron la tarea de construir un sector circular con GeoGebra. Radford (2006) argumenta que el aprendizaje "no consiste en construir o reconstruir el conocimiento. Se trata de dar sentido a los objetos conceptuales que el estudiante encuentra en su cultura" (p. 113). El aprendizaje sería entonces un proceso social, sensible y material teorizado en la TO como procesos de 
objetivación en la que "el estudiante alcanza una comprensión crítica, a través de la dotación de significados, objetos culturales matemáticos y su lógica cultural" (Radford, 2011, p. 45).

En oposición al paradigma individualista, la TO propone una reconceptualización del aprendizaje matemático, no como resultado de la acción del sujeto que construye su propio conocimiento, sino como "un proceso colectivo, cultural e históricamente situado que destaca el papel del trabajo social humano, el cuerpo, emociones y el mundo material" (Radford, 2018a). Para la TO, el aprendizaje tiene que ver con el saber matemático de la escuela, pero también con aquellos seres que se transforman y reafirman como sujetos de educación en la búsqueda de este conocimiento. Para estudiar el aprendizaje matemático, la TO introduce dos categorías conceptuales en forma de procesos de objetivación y subjetivación. Mientras que los procesos de objetivación explican la forma en que el conocimiento aparece en el aprendizaje, los procesos de subjetivación tienen que ver con el sujeto que hace parte de la actividad y sus formas de colaboración. En atención al objetivo de nuestro, nos referimos al aprendizaje matemático en términos de procesos de objetivación.

El cuanto al significado de objetivación en la TO, Radford (2018b, p.233), considera que la experiencia humana de las personas desde su nacimiento entra en contacto con situaciones, entidades o cosas que se les oponen, que parecen extrañas y desconocidas, pero que forman parte del repertorio de sistemas de expresión, acción y pensamiento, constituido histórica y culturalmente. La objetivación es precisamente este proceso social, corporal, material y simbólico que implica:

Llegar a ser, progresiva y críticamente, consciente de una forma codificada de pensamiento y acción, algo que gradualmente notamos y al mismo tiempo adquiere significado. Los procesos objetivos son aquellos actos para darnos cuenta significativamente de que algo se revela a la conciencia a través de nuestra actividad corporal, sensorial y de artefactos (Radford, 2017b, p. 121).

La forma de entender la objetivación propuesta por la TO revela dos elementos de este proceso que son fundamentales para comprender cómo se produce el aprendizaje geométrico durante la ESG. Por un lado, la objetivación es un proceso individual, emocional y afectivo de sensibilización sobre algo que constituye conocimiento. Por lo tanto, la conciencia es un reflejo de la forma en que cada individuo reconoce el mundo que lo trasciende y se orienta / posiciona 
críticamente dentro de él (subjetivación), dentro de una dinámica de encuentro dialéctico con las formas codificadas de reflexión, acción y pensamiento.

Esta conciencia no es contemplativa, porque, a través de la conciencia individual, se forman sensibilidades culturales para reflejar, comprender, estar en desacuerdo, objetar y sentir a los demás, a sí mismos y al mundo (Radford, 2017b, p. 122). Por otro lado, para que se produzca la conciencia, es necesario que los individuos se involucren en una determinada actividad, de modo que, las reflexiones y acciones de los estudiantes en ella conduzcan a formas de instanciación del conocimiento matemático que, al principio, puede parecer algo extraño para nosotros, como una especie de alteridad.

Durante los procesos de objetivación, los estudiantes y profesores usan el cuerpo, los signos, los artefactos y los gestos para hacer que el objeto aparezca (Sánchez, Prieto G., Gutiérrez \& Díaz-Urdaneta, 2020; Sánchez \& Prieto G., 2018; Sánchez \& Brandemberg, 2019) y alcanzar formas relativamente estables de conciencia acerca de los significados culturales con los que fueron dotados, ya que los objetos no pueden usarse al estar totalmente expuesto en el mundo concreto. Estos recursos que se utilizan en la actividad matemática se denominan medios semióticos de objetivación. En términos de Radford, los medios semióticos de objetivación se definen como:

Todos los medios utilizados por los individuos que están en el proceso de producir significados, para lograr una forma estable de conciencia, presentan sus intenciones y organizan sus acciones y así adquieren los objetivos de sus acciones (Radford, 2003, p. 41).

Es a través de la movilización de medios semióticos de objetivación en la actividad matemática escolar que se puede acceder a los objetos matemáticos, lo que les permite estar presentes, dándoles una cierta forma de conocimiento tangible y corporal (Radford, 2003). Arzarello (citado en Vergel, 2014) afirma que es necesario en la investigación detectar y analizar cómo surgen y evolucionan los medios semióticos en el proceso de enseñanza y aprendizaje de las matemáticas.

En la teoría de la objetivación, lo que hace posible el aprendizaje es una actividad humana, sensual y práctica. En este sentido, el concepto de actividad propuesto desde la TO cubre mucho más que las personas que interactúan entre sí. Es más que un medio de interactuar con personas y artefactos. Es una forma de vida, algo orgánico y sistémico, un evento creado 
por una búsqueda común, es decir, una búsqueda con otras personas, de la solución a un problema planteado, una investigación cognitiva, emocional y ética (Radford, 2017b, p. 125). Radford, para evitar confusiones con otros significados de la actividad, decidió llamar "trabajo conjunto" en la teoría de la objetivación a la actividad.

Radford coloca la labor conjunta como la principal categoría de la teoría de la objetivación. Se basa en las ideas de Spinoza (1989), donde "los seres humanos son considerados parte de la naturaleza: son seres naturales, seres de necesidades que encuentran su satisfacción en los objetos fuera de sí mismos" (Radford, 2018b, p. 12, traducción nuestra). Para satisfacer sus necesidades (necesidades de supervivencia, aprendizaje y otras necesidades creadas por / en la sociedad), los seres humanos se lanzan activamente al mundo. Durante esta producción los individuos se inscriben en el mundo social y al mismo tiempo producen su propia existencia. El nombre de este proceso Radford le llamó labor conjunta.

Colocar el trabajo conjunto como una categoría ontológica y epistemológica en la teoría de la objetivación nos lleva a considerar la actividad en el aula como la unidad de análisis (Radford, 2018a). Sin embargo, no podemos descartar el papel del lenguaje, los signos, los artefactos y el cuerpo en los procesos de objetivación. En la TO, el lenguaje, los signos, los artefactos y el cuerpo sensible se entienden no como mediadores, sino como parte de la actividad de los individuos, parte de su pensamiento.

Considerando un entorno escolar, esta búsqueda común se entiende precisamente como "lo que los maestros y los alumnos producen juntos en el aula, trabajando codo con codo (por ejemplo, una o más formas de presentar y / o resolver un problema, demostrar, etc.)" (Radford, 2020). Hegel (2001) se refiere a esta búsqueda a través de la expresión obra común, sobre dicha base Radford (2018b) establece lo siguiente:

Es en la producción de esta obra común que se encuentran los estudiantes con el saber cultural e histórico. Esta reunión consiste en una conciencia progresiva, material y sensible del conocimiento, por lo que el aprendizaje no es más que la refracción en la conciencia del estudiante de ese trabajo común que él o ella contribuyó a hacer aparecer en el aula (p. 14).

Teniendo en cuenta las ideas teóricas anteriores, definimos la Elaboración de Simuladores con GeoGebra (ESG) como un contexto en el que se producen varias actividades creadas por la necesidad de promover el aprendizaje matemático, en específico el geométrico, los involucrados que participan en esta actividad son motivados por la producción de dibujos 
dinámicos con el software GeoGebra. Un dibujo dinámico es un dibujo geométrico producido por medio de un software dinámico, con el fin de preservar las propiedades espaciales que se le impusieron cuando es movido o arrastrado por cualquiera de sus elementos libres (Laborde, 1997).

Para producir los dibujos dinámicos, los alumnos y profesores despliegan una secuencia de actividades (Castillo, Prieto G., Sánchez \& Gutiérrez, 2019; Gutiérrez \& Castillo, 2020; Sánchez \& Sánchez, 2020) donde se ponen de manifiesto diferentes tipos de obra común. Según Rubio, Prieto \& Ortiz (2016), el primer conjunto de actividades tiene como producto la traducción, en términos geométricos, del boceto de alguna parte que compone al fenómeno de la simulación. En este caso, la obra común se manifiesta en el modelo matemático producido durante la actividad y el establecimiento de una secuencia de construcciones con GeoGebra que son atendidas en las demás actividades. El segundo conjunto de actividades resulta en la producción del dibujo dinámico correspondiente al modelo matemático producido anteriormente (Gutiérrez, Prieto G., \& Ortiz, 2017). Usamos los términos matematización y trabajo matemático para referirnos al primer y segundo subtipo de actividades, respectivamente. En este trabajo, centraremos nuestra atención en el trabajo matemático realizado por unos estudiantes de pregrado y profesores para representar el sector circular en el GeoGebra.

Así, durante este tipo de actividades estudiantes y profesores: (i) seleccionan la herramienta o funcionalidad dinámica del GeoGebra que permite construir el objeto geométrico de la primera tarea de la secuencia (Castillo \& Prieto G., 2018), (ii) identifican en la vista gráfica la presencia o ausencia de cada elemento requerido por la herramienta o funcionalidad del software para construir el objeto geométrico en cuestión, (iii) construyen los elementos faltantes aplicando una estrategia determinada, (iv) aplican la herramienta o funcionalidad del GeoGebra, (v) validan la consistencia de la construcción por medio de la prueba del arrastre, y (vi) aplican nuevamente las acciones anteriores para los demás objetos identificados en el boceto, hasta producir el dibujo dinámico.

Es durante el desarrollo de estas actividades donde surgieron los procesos de objetivación de los objetos geométricos utilizados para representar le sector circular, colocando especial interés en los medios semióticos utilizados para expresar las ideas geométricas. 


\section{Marco Metodológico}

Para contestar la pregunta de esta investigación, se realizó un estudio cualitativo (Bogdan \& Biklen, 1994) de corte descriptivo y de campo (Fiorentini \& Lorenzato, 2012). En los siguientes sub-apartados se describen los participantes y el contexto de la investigación, colecta de información, así como la forma en la que los datos fueron producidos y analizados.

\section{Participantes y contexto}

Los participantes de esta investigación fueron 14 estudiantes de pregrado del grado integrado de Ciencias, Matemáticas e Idiomas en los Años Iniciales de la Escuela Primaria del Instituto de Educación Matemática y Científica (IEMCI), quienes participaron en la disciplina Tendencias de Investigación en Ciencias, Matemáticas e Idiomas I del tercer período. En algunas clases de esta disciplina, los estudiantes participaron en la actividad llamada "Construir dibujos dinámicos con GeoGebra" que se llevó a cabo en mayo y junio del año 2019. Para mantener el anonimato de los participantes de la investigación, los estudiantes serán llamados con el acrónimo $\mathrm{E}$, seguido de un número y los profesores participantes, como $\mathrm{P}$ con un número al lado.

Durante estas reuniones, se llevaron a cabo 4 actividades. La primera sesión tuvo como objetivo familiarizar a los jóvenes con la actividad de simuladores con GeoGebra. Con esto queremos decir que esta sesión consistió en presentar los procesos matemáticos asociados con la resolución de algún fenómeno, es decir, explicar cada momento que los estudiantes pasarán para desarrollar el simulador computacional. Luego, se realizó un primer acercamiento al software GeoGebra, donde se anunciaron los puntos más importantes, quién fue el creador, la comunidad que lo acompaña y los materiales disponibles para su uso en las aulas. A continuación, se presentó a los estudiantes la interfaz GeoGebra, las áreas que la componen y las cajas de herramientas de construcción que el software puede poner a disposición de los usuarios (ver Figura 1). 
Figura 1: Interfaz del GeoGebra

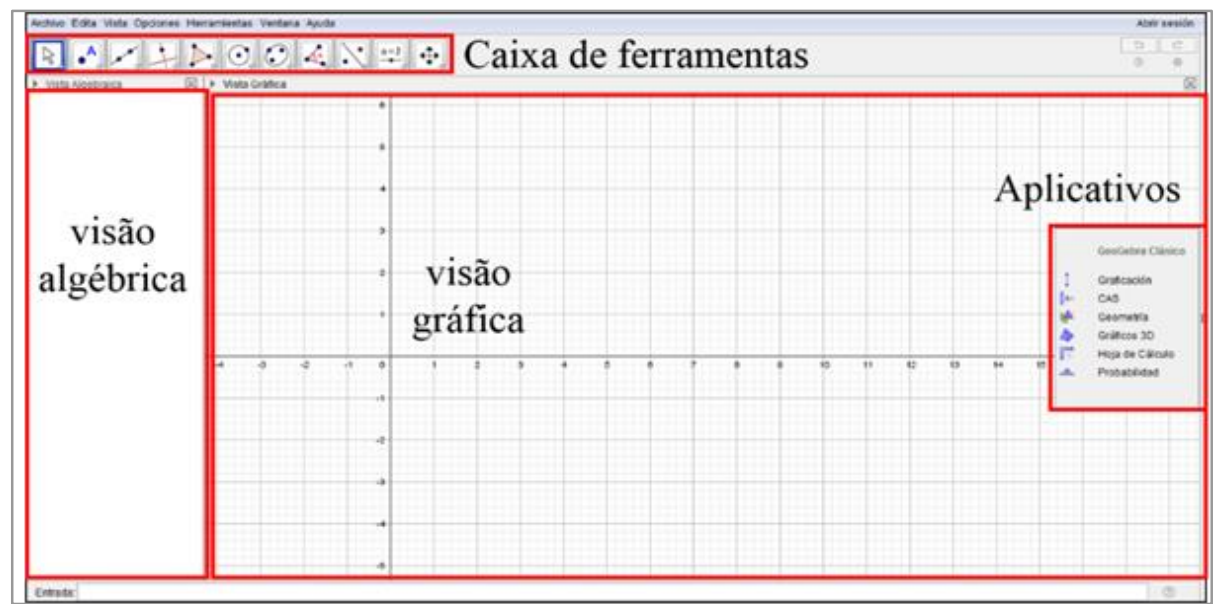

Fuente: Sánchez (2020)

La segunda y tercera sesión de trabajo estuvieron directamente relacionadas con el desarrollo de simuladores con GeoGebra. Por razones de tiempo, el fenómeno fue seleccionado por la persona responsable del taller. Este era un motor de dos tiempos. Después de toda la información sobre el fenómeno, los estudiantes simularon el cigüeñal en la visualización gráfica de GeoGebra (ver figura 2).

Figura 2: Imagen de referencia del fenómeno

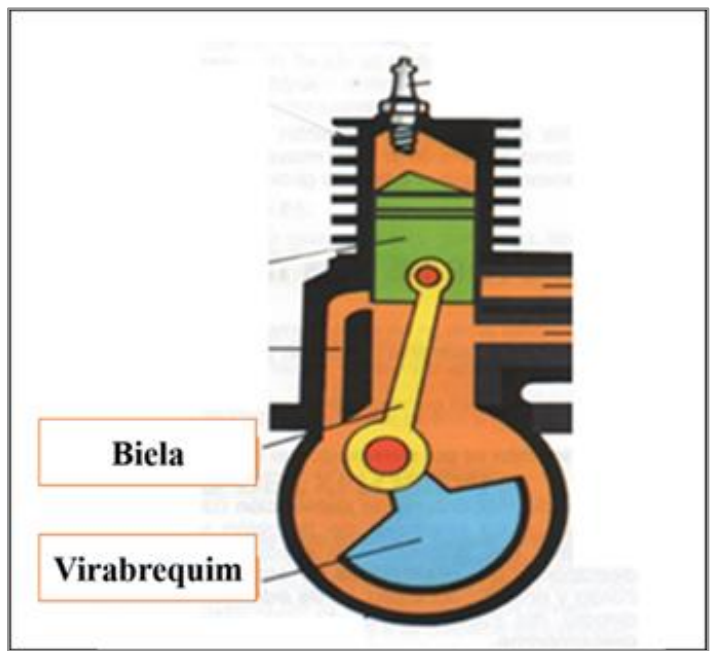

Fuente: Sánchez (2020)

\section{Recolección de datos}

Los datos del estudio provienen de la discusión generada por los participantes sobre la construcción del cigüeñal en GeoGebra. Esta discusión fue grabada con una cámara de video, para capturar la realidad natural y compleja del trabajo conjunto realizado por los participantes, 
al pasar por el "el trabajo matemático" descrita en las secciones anteriores. En el video, puede ver los recursos semióticos que los participantes usan para comunicar sus ideas. El video se transcribió en su totalidad en formato Word, utilizando un instrumento similar al que se muestra en la Tabla 1. La siguiente tabla muestra un ejemplo de cómo se transcribió la información de los videos grabados de las sesiones de trabajo.

Tabla 1: Instrumento para la transcripción de los episodios

\begin{tabular}{|c|c|}
\hline \multicolumn{2}{|c|}{$\begin{array}{l}\text { Momento. No.: } 1 \\
\text { Número de vídeo: } 1 \\
\text { Líneas: }(1-8) \\
\text { Discusión: Selección de piezas del fenómeno que tienen movimiento. }\end{array}$} \\
\hline $\begin{array}{l}\text { No. De } \\
\text { línea }\end{array}$ & Contenido de la transcripción \\
\hline 1 & $\begin{array}{l}\text { Profesor 1: ¿Cuántas piezas [que tienen movimiento] reconoces allí [en la pizarra]? Comparando las dos } \\
\text { imágenes [que se muestran en el cuadro]. }\end{array}$ \\
\hline 2 & E1: El pistón \\
\hline 3 & $\begin{array}{l}\text { Profesor } 1: \text { ¿Cuál sería el pistón? Podría suceder y hacer una señal a tus compañeros de equipo para que } \\
\text { te reconozcan. }\end{array}$ \\
\hline 4 & $\begin{array}{l}\text { E1: Para mí sería esta parte de aquí [mueve la mano hacia arriba y hacia abajo verticalmente (ver Figura } \\
\text { 3)]. } \\
\text { Figura 3. Gestos de un estudiante para explicar el movimiento de la pieza }\end{array}$ \\
\hline & Fuente: Sánchez (2020) \\
\hline 5 & Profesor 1: es este de aquí [apunta para una parte de la pizarra]. \\
\hline 6 & E1: Sí. \\
\hline 7 & Profesor 2: Ese es el pistón. \\
\hline 8 & E1: Para mi seria el pistón. \\
\hline
\end{tabular}

Fuente: Sánchez (2020)

\section{Análisis de los datos}

El análisis de los datos se realizó por etapas. En el paso 1, hicimos una primera lectura de las transcripciones para identificar, discutir y describir el proceso matemático utilizado por los estudiantes y profesores para representar el cigüeñal en el GeoGebra, así como también se reconoció la técnica de construcción implementada. El objetivo de esta etapa fue reconocer las 
particularidades del trabajo geométrico realizado durante el proceso mencionado, especialmente el contenido geométrico que se movilizó en esta actividad.

Tabla 2: Técnica de construcción del sector circular implementada por los estudiantes y profesores

Tarea de construcción: Construir un sector circular a partir de un punto exterior (punto A)

Herramienta utilizada: Sector Circular

Definición de la herramienta: Seleccione el centro y después, dos puntos.

Paso 1: Determinar los extremos del sector circular

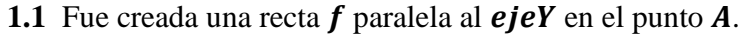

1.2 Fue creado un deslizador de tipo número, con valores mínimo y máximo de $\mathbf{1}$ y $\mathbf{5}$, respectivamente y un incremento de 0. 01. Este deslizador fue llamado padrón.

1.3 Fue creado un deslizador de ángulo $\boldsymbol{\alpha}$, com valores mínimo y máximo de $\mathbf{0}^{\circ}$ e $360^{\circ}$, respectivamente y un incremento de $\mathbf{1}^{\circ}$.

1.4 La recta $\boldsymbol{f}$ fue rotada con centro de rotación el punto $\boldsymbol{A}$, un ángulo $\boldsymbol{\alpha}$ y en sentido horario, generando la recta $\boldsymbol{f}^{\prime}$.

1.5 Fue creado un círculo $c$ con centro en el punto $A$ y radio igual a 1,08 * padrón.

1.6 Fue creado círculo $\boldsymbol{d}$ con centro en el punto $\boldsymbol{A}$ y radio igual a $0,45 *$ padrón.

1.7 La recta $\boldsymbol{f}$ 'fue interceptada con el círculo $\boldsymbol{d}$, generando los puntos $\boldsymbol{C}$ y $\boldsymbol{D}$.

1.8 Se creó una recta $\mathrm{g}$, perpendicular a la recta f ', que pasara por el punto $\mathrm{C}$.

1.9 Se intercepto la recta $\boldsymbol{g}$ con el círculo $\boldsymbol{c}$, generando os pontos $\boldsymbol{E}$ e $\boldsymbol{F}$.

Figura 4: Determinación de los extremos del sector circular

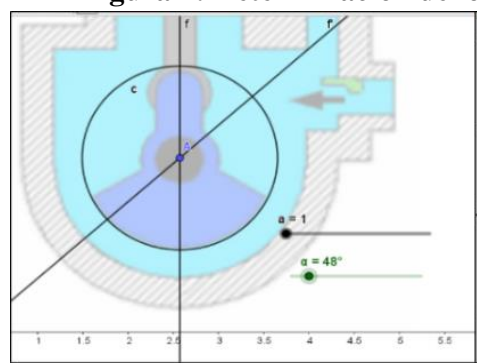

(a)

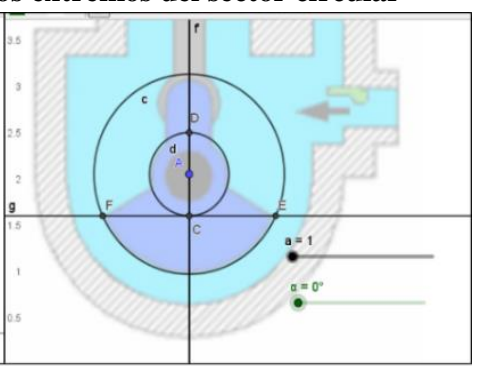

(b)

Fuente: Sánchez (2020)

Paso 2: Aplique la herramienta sector circular

2.1 Se creó el sector circular e de centro A y las de extremos E y F.

Figura 5: Construcción del sector circular

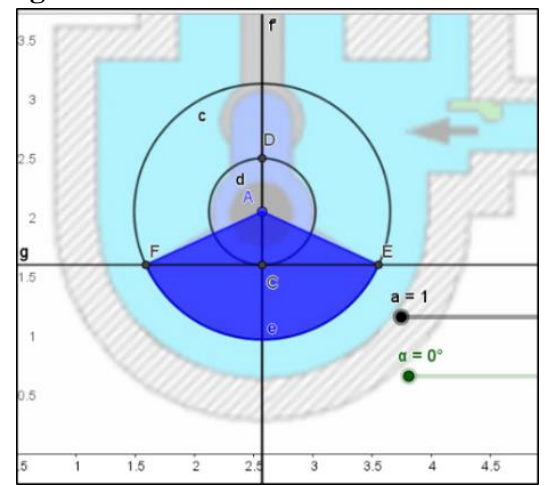

Fuente: Sánchez (2020)

Fuente: Sánchez (2020)

En el segundo paso, contactamos los datos recopilados. En este sentido, hicimos un recorrido por las transcripciones para detectar fragmentos de la discusión de los participantes 
que parecían mostrar evidencia de la conciencia de algún objeto geométrico subyacente durante el trabajo matemático. Cada fragmento es un segmento destacado (Radford, 2015, p. 561) que refleja un momento particular en el trabajo matemático para representar el cigüeñal. Para identificar los segmentos, nos enfocamos en los cambios de enfoque a lo largo de la discusión, considerando los objetos geométricos que identificaron a los participantes.

En la tercera etapa, realizamos un análisis multisemiótico (Radford, 2015; Sabena, Robutti; Ferrara \& Arzarello, 2012), con el fin de identificar en los segmentos la variedad de significados y medios semióticos realizados por los participantes durante el trabajo matemático. Asumiendo que la cognición tiene una naturaleza multimodal (Arzarello, 2006; Manghi, 2011; Radford, Edwards \& Arzarello, 2009), nos enfocamos en cómo los participantes combinaron diferentes signos y artefactos para tratar de hacer aparentes los objetos geométricos que pueden representar la parte del fenómeno a ser simulado.

Llamamos al nodo semiótico cada forma en la que los participantes combinaron diferentes medios semióticos para lograr un estado de conciencia más o menos estable sobre algún objeto geométrico. Radford (2003) define los nodos semióticos como "piezas de la actividad semiótica del estudiante, donde la acción, los gestos y las palabras trabajan juntos para lograr la objetivación del conocimiento" (p. 56).

En este análisis consideramos las formas de acción, expresión y reflexión asociadas con el desarrollo del aprendizaje geométrico para estudiantes de pregrado de pregrado integrados en la resolución de la construcción del sector circular con GeoGebra. Por lo tanto, dada la naturaleza de la investigación y la pregunta, el enfoque será la interacción discursiva de los estudiantes a través de las transcripciones de las grabaciones de video, así como las producciones de la actividad matemática registrada en el archivo GeoGebra y las notas generadas y por el investigador. De acuerdo con la perspectiva de la Teoría de Objetivación (Radford, 2006, 2013), el análisis de datos se basa en una concepción multimodal del pensamiento humano (Arzarello, 2006). Esta concepción multimodal del pensamiento humano establece que el análisis de datos debe tener en cuenta la relación entre los diferentes sistemas semióticos movilizados por los estudiantes en el curso de la actividad (el sistema semiótico de lenguaje escrito, lenguaje hablado, gestos, acciones, etc.). Es por esta razón que ni la escritura, ni el habla, ni la gestión de los estudiantes se analizan de forma aislada, por el contrario, estas 
formas de reflexión, expresión y acción se estudian como partes constitutivas de los procesos de objetivación.

\section{Resultados}

El análisis de los datos permitió detectar segmentos destacados que se dieron cuenta de cómo los estudiantes y profesores, apoyados por una variedad de medios semióticos de objetivación, dieron cuenta de la toma de conciencia progresiva sobre la representación del sector circular en el GeoGebra. En total, hay dos nodos semióticos que representan diferentes objetos geométricos que surgieron durante el trabajo conjunto.

\section{Trabajo matemático}

A partir de las actividades desarrolladas durante el trabajo matemático por los estudiantes y profesores, se identificaron dos nodos semióticos que explican la discusión en torno a un objeto geométrico que se construirá en el sector circular. Podríamos decir que estos nodos están en correspondencia con los pasos y acciones de la técnica de construcción mostrada en la sección de metodología. La objetivación de estudiantes y docentes en dichos objetos geométricos responde a la conceptualidad de la herramienta en la construcción de un sector circular.

\section{Nodo 1. La idea de rotación}

El nodo comienza cuando la discusión entre profesores y alumnos gira en torno al reconocimiento de cuál objeto matemático puede representar el movimiento de la pieza del cigüeñal. El profesor 1, en un primer intento, para que los estudiantes se den cuenta de este objeto, utiliza el marcador en sus manos para simular el movimiento que está haciendo la pieza, al mismo tiempo, solicita a los jóvenes que identifiquen qué movimiento están simulando.

La respuesta de algunos estudiantes parece correcta, pero no tienen el foco de atención que el profesor 1 buscó cuando les pidió a los estudiantes que enfocaran su atención en algún objeto matemático; por lo tanto, con ayuda de su discurso oral va direccionando sus preguntas y sugestiones. Lo anterior y el constante movimiento de la mano del profesor 1 hace que E8 sepa que este movimiento puede representarse mediante una rotación. El profesor 1 considera 
al alumno cuando afirma que el movimiento del sector circular se puede representar en GeoGebra mediante una rotación.

112. Profesor 1: Ahora, imaginemos que el marcador que tengo en mi mano es el cigüeñal, qué movimiento estás haciendo en este momento [mueve el marcador en tu mano].

Figura 6: Simulación del movimiento del cigüeñal usando el marcador

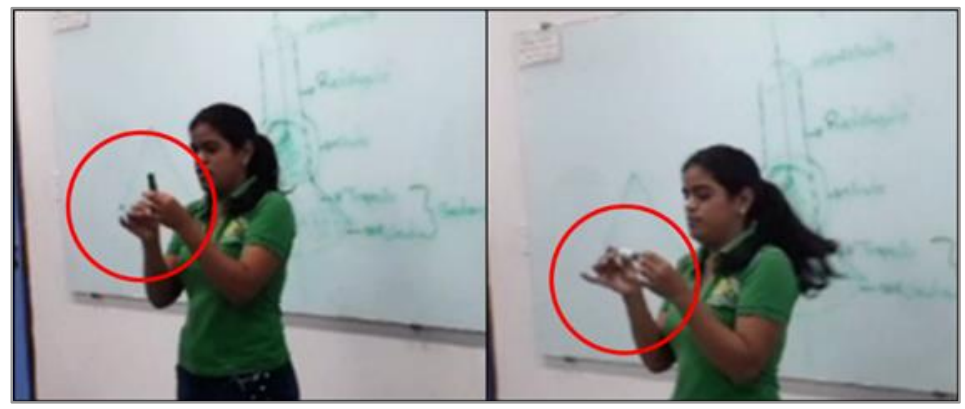

Fuente: Sánchez (2020)

113. E3, E6, E5 y E7: Está girando

114. Profesor 1: Está girando, es verdad. Pero si pensamos en cómo representar este giro matemáticamente, ¿cómo podemos hacerlo? Recuerdan las transformaciones de las que hablamos. Simetría axial...

115. E8: Con una rotación

116. Profesor 1: Es cierto que podemos simular el movimiento del sector circular con una rotación.

Para que los estudiantes se den cuenta de la idea de la rotación, deben considerar la conceptualidad detrás de la herramienta Rotación. Es decir, para considerar que los estudiantes aprendieron sobre la idea de la rotación, tuvieron que reconocer qué objeto rotar, el centro de rotación y el ángulo de rotación. Elementos necesarios para usar la herramienta, en otras palabras, para poder realizar la rotación. En este sentido, los estudiantes pudieron identificar, sin problemas, que el objeto que se rotaría es la recta ya dibujada en la vista gráfica. Del mismo modo, no tuvieron problemas para comunicar que el centro de rotación sería el punto A (punto previamente dibujado en el GeoGebra). Sin embargo, al identificar el ángulo de rotación, los estudiantes tuvieron problemas para reconocer que este valor sería variable ya que, E3 comenta que el ángulo de rotación es de $360^{\circ}$ (línea 134),

El profesor 2 aprovecha la respuesta de este estudiante para mostrarle con la ayuda de GeoGebra que no es posible considerar un solo ángulo en esta ocasión, sino más bien una familia de ángulos que se pueden representar con la herramienta deslizante y que tendrían un valor mínimo de $0^{\circ}$ y un valor máximo de $360^{\circ}$. Después de esta explicación, el Profesor 1 mostró la 
rotación ahora considerando un deslizador de ángulo $\alpha$ con los valores mencionados, obteniendo la recta necesaria.

127. Profesor 2: La herramienta de rotación solicita el objeto a rotar, el centro de rotación y un ángulo de rotación. ¿Cuál sería el objeto a rotar?

128. E4: La recta que dibujamos

129. Profesor 2: Bien, ¿qué pasa con el centro de rotación? Deben considerar los objetos que se crean en la vista gráfica.

130. E6: Entonces sería el punto A, que es el único punto allí

131. Profesor 2: ¿Y cuál sería el ángulo de rotación?

132. E3: ¿Entonces tenemos que crear una recta encima de la que ya está dibujada, pero que esta tenga movimiento?

133. Profesor 2: Así es

134. E3: Bueno, si estuviera pensando en que la recta tenga movimiento, sería $360^{\circ}$

135. Profesor 2: Bueno, el profesor 1 hará la rotación, hará clic en la recta, luego en el punto A y luego, en la ventana emergente, colocará $360^{\circ}$. A continuación, tenemos una recta $f^{\prime}$, que se rotó $360^{\circ}$ [Figura 7].

Figura 7: El profesor apunta la recta rotada con su mano

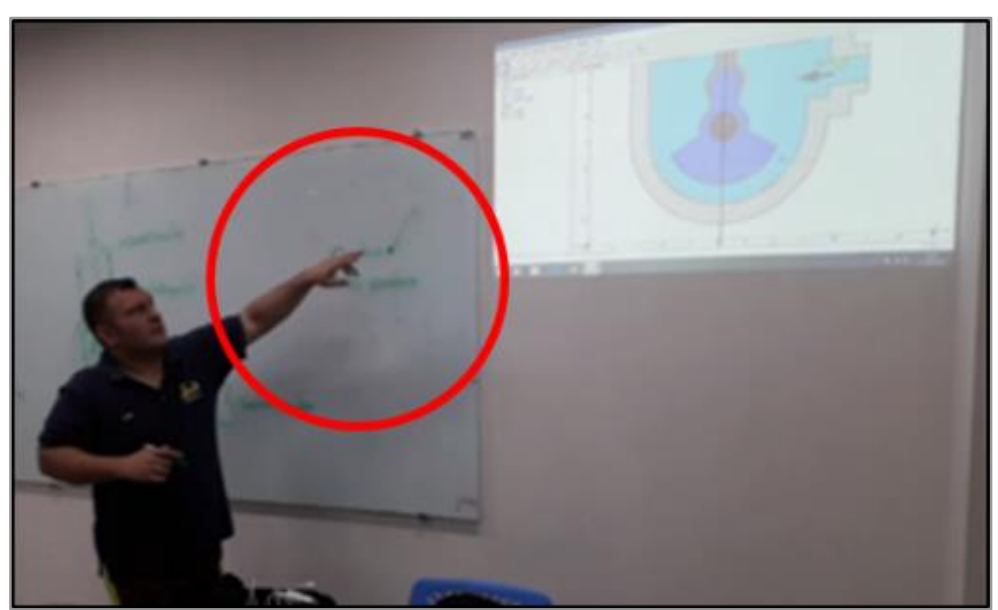

Fuente: Sánchez (2020)

136. Profesor 2: Pero esta rotación no nos ayuda porque no se mueve, necesitamos hacer una rotación con un ángulo dinámico, que varía de $0^{\circ}$ a $360^{\circ}$. En GeoGebra, podemos hacer esto creando un deslizador.

137 Profesor 1: Ya creé el deslizador, con un valor mínimo de $0^{\circ}$ y un máximo de $360^{\circ}$. Ahora rotaré la recta f, en relación con el punto A y en el valor del ángulo, pondré el nombre del deslizador. Ahora podemos ver la rotación de la recta. 
Figura 8: Rotación de la recta f en relación al punto $\mathrm{A}$ y ángulo $\alpha$

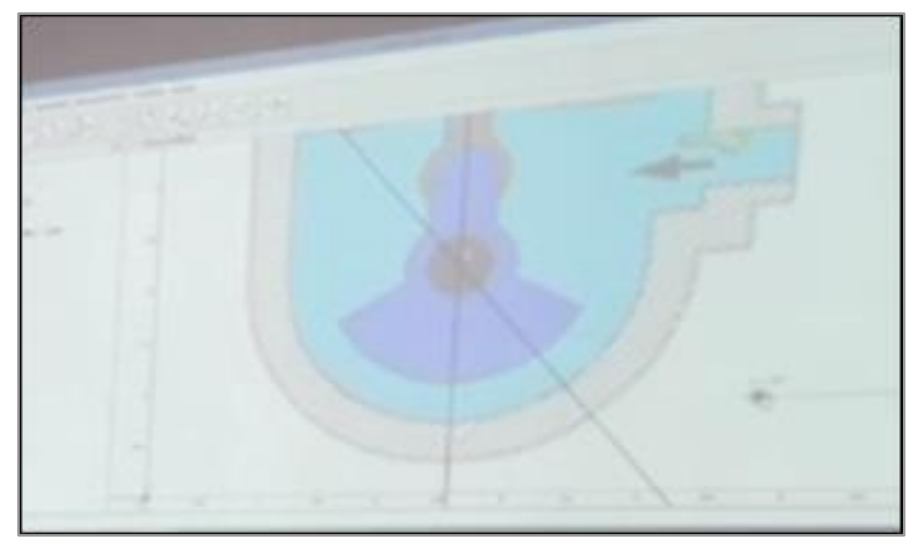

Fuente: Sánchez (2020)

138. E1, E9, E7, E8, y E3: ¡Que bien!

139. E7: ¿Entonces esa es la recta que vamos a utilizar? ¿La rotada?

140. Profesor 1: Así es, vamos a ubicar los extremos del sector circular en esa recta.

El uso del GeoGebra para mostrar que el ángulo de rotación no podía ser fijo sino variable fue de gran ayuda para que los estudiantes tomarán conciencia de que no necesitaban un solo ángulo, como estaban pensando en un inició, sino un conjunto de ángulos los cuales ayudarían a simular que la recta siempre está en movimiento.

\section{Nodo 2. los extremos del sector circular}

El trabajo conjunto en este punto de la discusión tiene como objetivo determinar la ubicación de los extremos del sector circular. El profesor 2 comienza la discusión comentando que estos puntos también deben tener movimiento, tal como la línea recta ya rotada. Estos puntos no pueden construirse en cualquier parte del plano, su ubicación debe estar relacionada con la recta rotada. El profesor 2, con la ayuda de las manos, muestra a los estudiantes cómo debe ser el movimiento de los puntos e indica con sus manos dónde deben ubicarse. Este maestro deja una pregunta a los estudiantes sobre cómo pueden determinar estos puntos, considerando el uso de la regla y el compás.

104. Profesor 2: Entonces, si ya tenemos uno de los puntos solicitados por la herramienta, que es el centro, ¿cuáles son los otros dos puntos que necesito?

105. E2: Los extremos.

106. Profesor 2: Exactamente, así que sé que debemos construir los extremos, que exactamente se ubicarán aquí [señala con la flecha del mouse] y aquí [señala una parte en la pantalla]. Pero esos dos puntos no puedo construirlos haciendo clic y haciendo clic.

107. E3: Tal como se hizo el punto anterior. 
108. Profesor 2: Sí. Estos [puntos deben construirse con otras construcciones auxiliares] y también que proporcionan este dinamismo [mueve una de sus manos en un círculo, Figura 9].

Figura 9: El profesor 2 simula el movimiento circular con el dedo

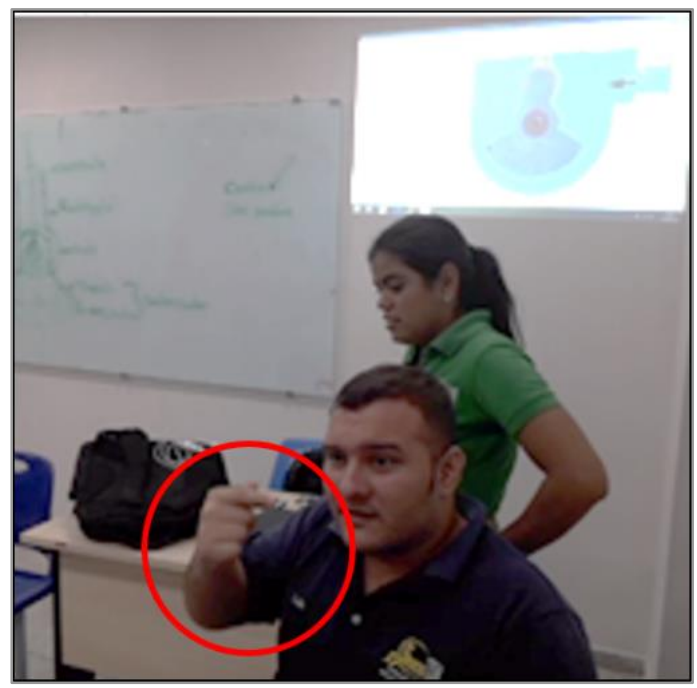

Fuente: Sánchez (2020)

109. E3: Para realizar este movimiento circular...

110. Profesor 1: Porque si ya tenemos un punto que tiene movimiento [para simular el movimiento circular], entonces lo que tengo que hacer es realizar las demás construcciones en función de ese punto.

111. Profesor 1: Bueno, debemos ubicar [en GeoGebra] estos puntos [ver los extremos del sector circular dibujado en la pizarra, Figura 10a]. Estos puntos tienen este movimiento [con la ayuda de un marcador, hace el movimiento que tendría que tener los puntos, Figura 10b]. Luego debemos hacer otras construcciones para determinar estos puntos.

\section{Figura 10: La profesora 1 apunta los extremos del sector circular en la pizarra y el movimiento que} debería tener

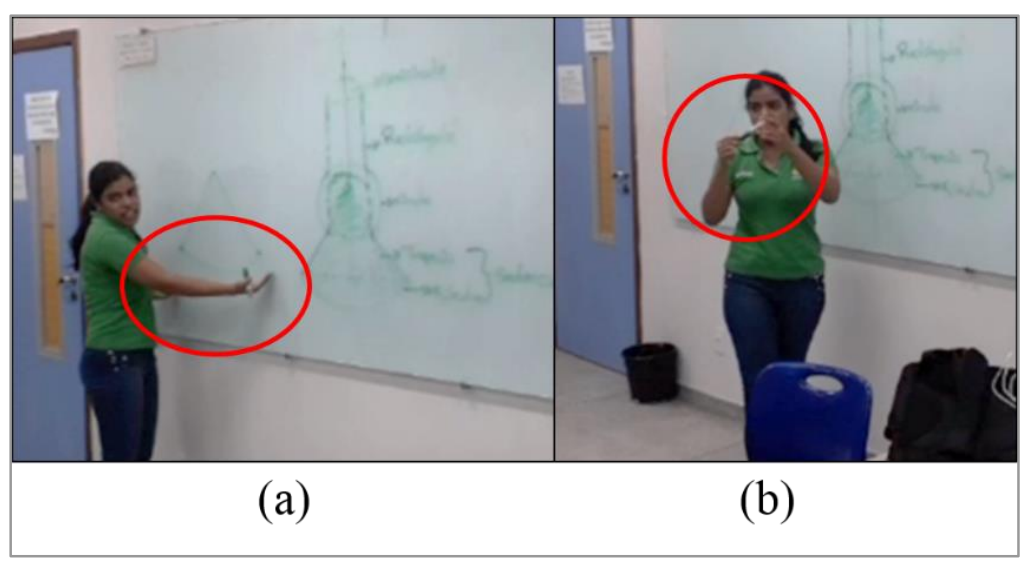

Fuente: Sánchez (2020)

139. Profesor 1: Ahora, desde esta recta, necesitamos determinar este punto [señala el punto con el dedo índice] y este punto [señala el punto con el dedo índice]. 
Figura 11: La profesora apunta la localización de los extremos del sector circular

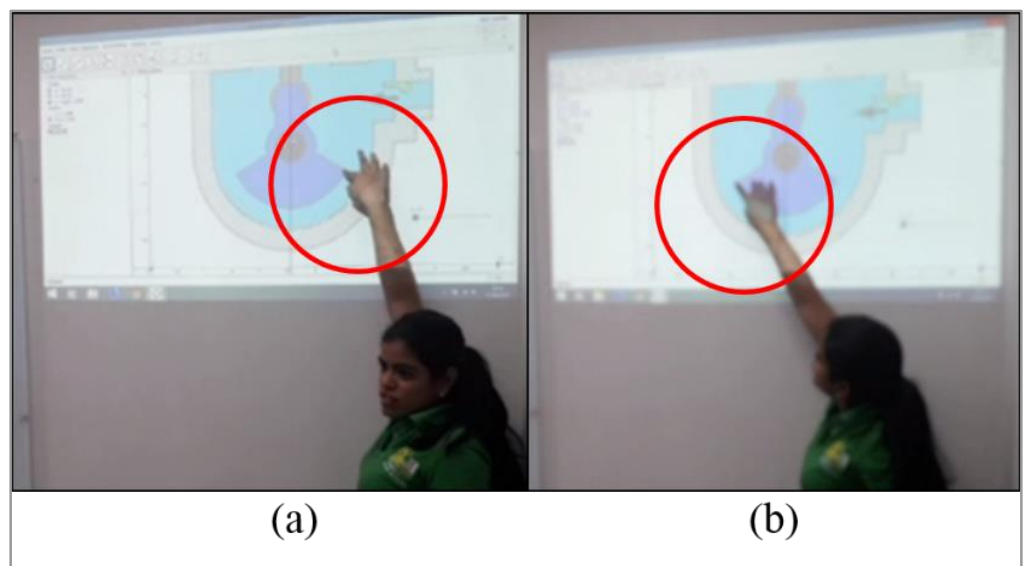

Fuente: Sánchez (2020)

140. Profesor 1: Ahora, si veamos el dibujo en la pizarra. Tenemos esta recta ahora [dibuje la línea, Figura 12a]. ¿Cómo determino este punto [señala el punto con la mano, Figura 12b] y ese punto [señala el punto con la mano, Figura 12c], usando una regla y compas?

Figura 12: Dibuja la recta y apunta las extremidades del sector circular

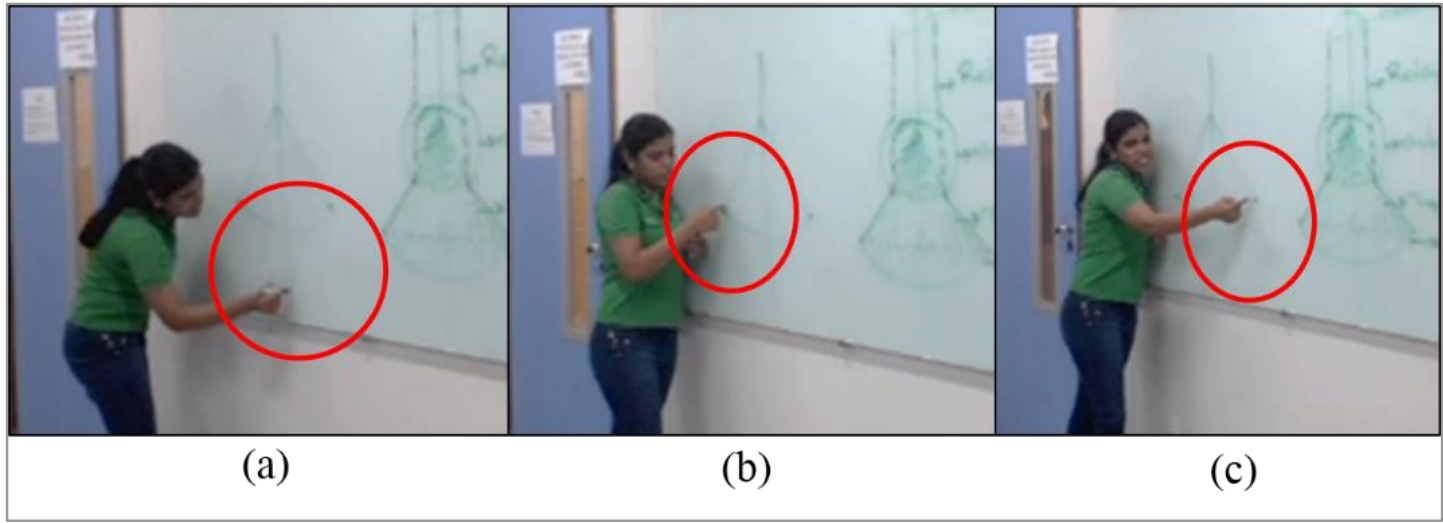

Fuente: Sánchez (2020)

E9 decide participar y mostrar a sus compañeros de equipo cómo determinaría estos puntos. Para esto, el estudiante se basa en el dibujo para dibujar un círculo que pase por donde deben ubicarse los extremos. Luego, se apoya nuevamente en el dibujo para trazar una recta perpendicular a la recta f '(línea rotada). Sin embargo, al no incluir en su discurso cómo determino esta recta, hace que el profesor 1 intervenga y le pregunte cómo dibujó esa recta, ya que primero debe saber el punto por donde pasa la recta.

El estudiante E9 muestra signos de confusión, permanece en silencio y al final no da una respuesta a la profesora 1; por lo tanto, su compañero E3 decidió intervenir para ayudar, explicando que se puede crear un círculo centrado en el punto A y que llega al punto donde debe 
pasar la recta. Los profesores están de acuerdo con la técnica de construcción discutida y deciden hacerlo en GeoGebra para construir el sector circular y resolver la tarea.

141. E9: Primero, haría un círculo centrado en el punto A y pasaría por los extremos del sector circular [dibuje el círculo].

Figura 13: E9 dibuje el círculo

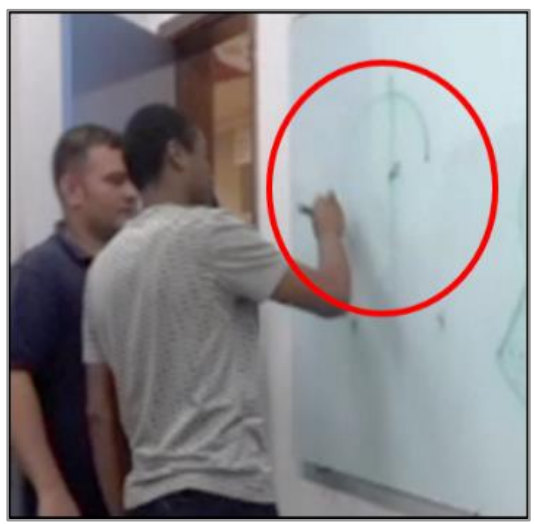

Fuente: Sánchez (2020)

142. Profesor 2: Bueno, sé que esos puntos están en ese círculo. Ahora, ¿cómo los determino? 143. E9: Crearía otra recta aquí [dibuje la recta, Figura 48]. la línea cruza la circunferencia y luego determino los puntos.

Figura 14: E9 dibuja la recta perpendicular

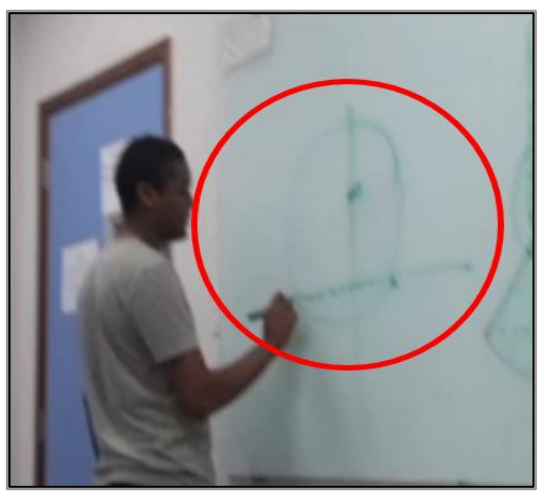

Fuente: Sánchez (2020)

144. Profesor 1: Pero ¿cómo hiciste eso? Porque primero debes ubicar un punto y luego dibujar la recta que debe estar relacionada con la que ya se ha creado. Creo que la recta que dibujaste es perpendicular a la que ya tenemos.

145. E3: Podemos crear otro círculo cuyo centro es el punto A y llega al punto donde pasa la recta perpendicular [E3 dibuja un círculo, Figura 15]. 
Figura 15: E3 dibuja un círculo

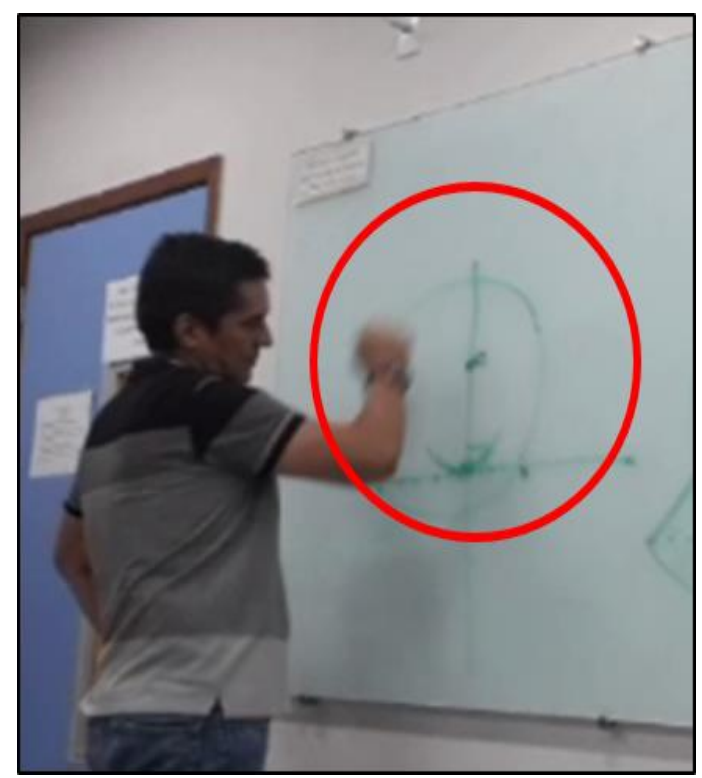

Fuente: Elaborada por los autores

146. Profesor 1: Bien, con eso podemos determinar los extremos del sector circular. Ahora llevemos lo que hemos discutido aquí a GeoGebra.

\section{Discusiones y Conclusiones}

En esta investigación, nos apoyamos en la perspectiva histórico-cultural del aprendizaje de las matemáticas, la teoría de objetivación da cuenta de los procesos de objetivación en torno a la noción del sector circular movilizados por estudiantes y profesores de matemáticas durante la construcción de un cigüeñal con GeoGebra. A través de un análisis multisemiótico, pudimos identificar algunos nodos semióticos que nos proporcionan información sobre tales procesos de objetivación del conocimiento geométrico que ocurrieron en las actividades de trabajo matemático. Estos hallazgos están relacionados con la actividad semiótica mostrada.

\section{La actividad semiótica exhibida}

Los resultados revelaron la variedad de signos (palabras, gestos, dibujos) y artefactos (cigüeñal, lápiz, papel, pizarra y el GeoGebra) que conforman los diferentes nodos semióticos identificados en las actividades de trabajo matemático. Con respecto a los gestos y los dibujos, identificamos que estos recursos desempeñaban un papel mediador importante en los procesos de objetivación, mientras que se producían de manera sincronizada con el discurso oral para revelar las ideas geométricas durante la actividad de trabajo matemático y la conceptualidad 
geométrica implícita en la herramienta Sector Circular utilizada por los estudiantes. El conjunto de signos y sus relaciones en cada momento de la discusión es un ejemplo de lo que Arzarello (2006) llama el paquete semiótico. Dentro del alcance de la actividad semiótica utilizada en los nodos semióticos, los gestos fueron utilizados por estudiantes y profesores, tanto para indicar los objetos geométricos necesarios para construir el sector circular en el GeoGebra como para recrear ciertos movimientos relacionados con la pieza.

\section{Medio semiótico kinestésico}

Las señas emitidas por estudiantes y profesores tienen como objetivo mostrar el objeto geométrico al que se refieren. Esta señalización puede ser para indicar la ubicación en el dibujo de algún objeto geométrico, alguna herramienta GeoGebra o algún escrito en la pizarra. También puede indicar alguna parte del fenómeno que se ha simulado. A lo largo de las evidencias mostradas en los resultados, se observa que este es uno de los medios semióticos más utilizados durante el trabajo matemático. El medio semiótico de señas, fue usado para indicar las ubicaciones del centro del sector circular, sus extremos, rectas, círculos entre otros.

Los movimientos, que representan este movimiento en el aire o en alguna superficie, en papel, en la pizarra o en el GeoGebra, también fueron utilizados por maestros y estudiantes. Este desplazamiento estaba relacionado con la forma de algún objeto geométrico, como fue el caso los profesores, quienes este medio kinestésico para indicar el movimiento circular del sector circular y la dirección de una recta perpendicular.

Las inscripciones realizadas por estudiantes y profesores durante las actividades de trabajo matemático fueron otro medio semiótico. Esto se caracteriza por dejar un registro (que se puede escribir o dibujar) de lo que se considera relevante durante el desarrollo de la actividad. Como fue el caso cuando se escribió en la pizarra los elementos que requiere la herramienta del sector circular para ser utilizada.

Los dibujos realizados por estudiantes y profesores también se consideran medios semióticos de objetivación, en la medida en que permiten el acceso o funcionan como una representación de objetos geométricos, relaciones geométricas, entre otros. Su uso les permite llevar al plano material lo que no se puede expresar con palabras o gestos y que desean mostrar a otros participantes en la actividad. Estos dibujos son signos utilizados intencionalmente para 
mostrar, por ejemplo, la dirección de una recta, como fue el caso del profesor. O tal como lo hizo E9 también quien dibujó círculos y rectas durante su explicación para determinar los extremos del sector circular.

\section{Medios semióticos lingüísticos}

Entre las expresiones lingüísticas utilizadas por estudiantes y profesores para complementar y sincronizar los gestos espaciales, había algunas expresiones lingüísticas como "esta", "allí" o "aquí". Los estudiantes lo usan para indicar un objeto geométrico específico que se dibuja en la pizarra. También "aquí" se utiliza para indicar la ubicación de algún objeto geométrico, como, por ejemplo, la ubicación del centro y los extremos del sector circular y todas las figuras geométricas identificadas por los estudiantes en el dibujo de la a pizarra.

\section{Medios semióticos físicos y tecnológicos}

Durante las actividades de trabajo de matemático, los estudiantes y profesores usaron la pizarra para hacer un dibujo referente al sector circular y los pasos de la técnica que se utilizó. También durante estas actividades, utilizaron GeoGebra como recurso tecnológico para representar los objetos geométricos identificados.

Este tipo de medios semióticos identificados en nuestro análisis coincide con los reportados en otras investigaciones. En primer lugar, McNeill (1992) caracteriza el uso de las manos (acompañadas de un lápiz o no) para indicar o señalar cualquier parte del boceto, localizar puntos, líneas, curvas o ángulos en el dibujo a mano alzada y también para simular el movimiento de algunas partes del fenómeno, en dos categorías llamadas gestos icónicos y deitíticos. Siguiendo a este autor, podemos concluir que la dimensión icónica del trabajo conjunto estaba presente, porque para estudiantes y maestros algunos gestos eran visualmente similares a las entidades que pretendían describir. Específicamente, resalta una escena del primer nodo semiótico cuando el profesor 1 hace un movimiento de su mano en alusión a la transformación de rotación.

Otra dimensión identificada es la deítica, que se refiere a la forma en que los sujetos indicaron un objeto geométrico específico (o algunos de sus aspectos característicos) representados en el espacio del artículo. Sobre esta última categoría pudimos observarla durante el nodo 2 cuando los estudiantes señalaban en la pizarra las rectas y puntos dibujados. Ambas 
dimensiones se reportaron en los estudios de Gómez (2013) y Pantano (2014), aunque con diferentes nombres. En el caso de Gómez, los incluye en una categoría llamada señales kinestésicas. Por otro lado, Pantano es más específico cuando se refiere a ellos como signos de dedo o lápiz.

Los diseños también fueron recurrentes durante la construcción del sector circular. Según Laborde (1997), la interpretación de dibujos en geometría es una práctica que favorece el reconocimiento de las propiedades espaciales asociadas con las propiedades geométricas del objeto que el dibujo intenta modelar. En nuestra investigación, descubrimos que los maestros hicieron grandes esfuerzos para dirigir la discusión hacia los puntos de encuentro entre lo visual y lo teórico, asegurando que cada toque que conforma el dibujo signifique cierta calidad de un objeto geométrico.

Destacamos que, en investigaciones anteriores centradas en la matematización en actividades de ESG (Sánchez y Brandemberg, 2019; Sánchez y Prieto G., 2018), se ha podido evidenciar cómo el uso consciente y crítico de estos medios semióticos de objetivación por parte de los involucrados en la ESG dan cuenta de las formas de producción del conocimiento geométrico, de ese encuentro con el saber en términos de Radford (2014, 2020).

Finalmente, reconocemos que, los resultados de esta investigación representan un avance en la comprensión de los procesos de objetivación producido durante las actividades de trabajo matemático alrededor de la representación de un sector circular en el GeoGebra, consideramos que analizar una actividad específica de este tipo no garantiza un reconocimiento profundo de este fenómeno, por lo que necesitamos llevar a cabo otros estudios centrados en los procesos de objetivación que ocurren durante el trabajo matemático en la ESG, para que los resultados obtenidos contribuyan a que nuestros profesores mejoren las condiciones de gestión de sus actividades.

\section{Agradecimientos}

Este artículo se realizó con el apoyo de la Coordenação de Aperfeiçoamento de Pessoal de Nível Superior - Brasil (CAPES) - Código de Financiamento 001. 


\section{Referencias}

Arzarello, F. (2006). Semiosis as a multimodal process. Revista Latinioamericana de Investigación en Matemática Educativa, Número Especial sobre Semiótica, Cultura y Pensamiento Matemático, 267- 299.

Bogdan, R., \& Biklen, S. K. (2007). Qualitative Research for Education. Na Introduction to Theory and Methods (5. ${ }^{\mathrm{a}}$ ed.). Pearson.

Bogdan, R. C., \& Biklen, S. K. (1994). Investigação qualitativa em educação: Uma introdução à teoria e aos métodos (M. J. Alvarez, S. B. Santos e T. M. Baptista, Trad.). Porto Editora.

Gutiérrez, R. E., \& Castillo, L. A. (2020). Simuladores con el software GeoGebra como objetos de aprendizaje para la enseñanza de la física. Tecné, Episteme Y Didaxis: TED, (47). https://doi.org/10.17227/ted.num47-11336

Castillo, L. A., \& Prieto G., J. L. (2018). El uso de comandos y guiones en la elaboración de simuladores con GeoGebra. UNION, 52, 250-262.

Castillo, L. A., Prieto G., J. L., Sánchez, I. C., \& Gutiérrez, R. E. (2019). Uma experiência de elaboração de um simulador com GeoGebra para o ensino do movimento parabólico. Paradigma, 40(2), 196-217. 10.37618/PARADIGMA.1011-2251.2019.p196-217.id764

Castillo, L. A., \& Sánchez, I. C. (2020). As formas de colaboração humana durante a elaboração de um simulador com o GeoGebra. O caso de David e Carolina. Revista Thema. 17(3), 572-583. https://doi.org/10.15536/thema.V17.2020.572-583.1110

Fiorentini, D. \& Lorenzato, S. (2012). Investigação em Educação Matemática: Percursos teóricos e metodológicos (3a edição). Autores Associados. (Original publicado em 2006).

Gómez, J. (2013). La generalización de patrones de secuencias figurales y numéricas: Un estudio de los medios semióticos de objetivación y procesos de objetivación en estudiantes de grado décimo [Tesis de maestría no publicada, Universidad Pedagógica Nacional de Colombia].

Gutiérrez, R. E., Prieto G., J. L., \& Ortiz, J. (2017). Matematización y trabajo matemático en la elaboración de simuladores con GeoGebra. Educacion Matematica, 29(2), 37-68. https://doi.org/10.24844/EM2902.02

Hegel, G. (2001). The philosophy of history. Kitchener. (Original publicado en 1837).

Laborde, C. (1997). Cabri-Geómetra o una nueva relación con la Geometría. In: Puig, L. (Ed.). Investigar y enseñar. Variedades de la educación matemática, (pp. 33-48). Una Empresa Docente.

Manghi, D. M. (2011). La perspectiva multimodal sobre la comunicación: desafíos y aportes para la enseñanza en el aula. Diálogos educativos, 22, 3-14.

Mcneill, D. (1992). Hand and mind: What gestures reveal about thought. Chicago, IL: University of Chicago Press. 
Pantano, O. (2014). Medios semióticos y procesos de objetivación en estudiantes de tercer grado de primaria al resolver tareas de tipo aditivo en los naturales. [Tesis de maestría no publicada, Universidad Pedagógica Nacional de Colombia].

Prieto G., J. L., \& Ortiz, J. (2019). Saberes necesarios para la gestión del trabajo matemático en la elaboración de simuladores con GeoGebra. Bolema: Boletim de Educação Matemática, 33(65), 1276-1304. https://doi.org/10.1590/1980-4415v33n65a15

Prieto G., J. L., Castillo, L. A., \& Márquez, M. (2020). Formas de colaboración humana entre profesores y alumnos durante la elaboración de simuladores con GeoGebra. Bolema: Boletim de Educação Matemática, 34(66), 199-224. https://doi.org/10.1590/19804415v34n66a10

Radford, L. (2003). Gestures, speech, and the sprouting of signs: A semioticcultural approach to students' types of generalization. Mathematical Thinking and Learning, 51(1), 37-70.

Radford, L. (2006) Elementos de una teoría cultural de la objetivación. Revista Latinoamericana de Investigación n Matemática Educativa, 9(Especial), 103-129.

Radford, L. (2011). The evolution of paradigms and perspectives in research. The case of mathematics education. In J. Vallès, D. Álvarez, \& R. Rickenmann (Eds.). Teacher's activity: Intervention, innovation, research, (pp. 33-49), Spain.

Radford, L. (2013). Three Key Concepts of Theory of Objectification: Knowledge, Knowing, and Learning. Journal of Research in Mathematics Education, 2(1), 7-44.

Radford, L. (2014). De la teoría de la objetivación. Revista Latinoamericana de Etnomatemática, 7(2), 132-150.

Radford, L. (2015). Methodological aspects of the theory of objectification. Revista Perspectivas da Educação Matemática, 8(18), 547-567.

Radford, L. (2017a). Saber y conocimiento desde la perspectiva de la Teoría de la Objetivación. En B. D’Amore \& L. Radford (Eds.), Enseñanza y aprendizaje de las matemáticas: problemas semióticos, epistemológicos y culturales, (pp. 115-136). Universidad Distrital Francisco José de Caldas.

Radford, L. (2017b). Aprendizaje desde la perspectiva de la teoría de la objetivación. En B. D’Amore \& L. Radford (Eds.), Enseñanza y aprendizaje de las matemáticas: problemas semióticos, epistemológicos y culturales, (pp. 97-112). Universidad Distrital Francisco José de Caldas.

Radford, L. (2018a). Algunos desafíos encontrados en la elaboración de la teoría de la objetivación. PNA, 12(2), 61-79.

Radford, L. (2018b, 27-29 de junho). Saber, aprendizaje y subjetivación en la teoría de objetivación [Palestra]. $\quad 5^{\circ}$ Simpósio Internacional de Pesquisa em Educação Matemática, Belém, Brasil. SBEM-PA.

Radford, L. (2020). Un recorrido a través de la teoría de la objetivación [A journey through the theory of objectification]. In S. Takeco Gobara \& L. Radford (Eds.), Teoria da Objetivação: Fundamentos e aplicações para o ensino e aprendizagem de ciências e matemática (pp. 15-42). Livraria da Física. 
Radford, L., Edwards, L. \& Arzarello, F. (2009). Introduction: Beyond words. Educational Studies in Mathematics, 70(3), 91-95.

Sabena, C., Robutti, O., Ferrara, F., \& Arzarello, F. (2012). The development of a semiotic frame to analyse teaching and learning processes: Examples in preand post-algebraic contexts. En L. Coulange; J-P. Drouhard; J-L. Dorier; A. Robert (Eds.), Recherches en Didactique des Mathématiques, Numéro spécial hors-série, Enseignement de l'algèbre élémentaire: bilan et perspectives, (pp. 231-245). La Pensée Sauvage.

Sánchez, I. C. Aprendizagem geométrica em torno das ideias presentes na simulação de um motor a dois tempos no GeoGebra. Um estudo de caso. 2020 [Dissertação de Mestrado, Universidade Federal do Pará].

Sánchez, I. C., \& Brandemberg, J. C. (2019). Aprendizagem geométrica e semiótica na matematização Com GeoGebra: O caso do virabrequim. REMATEC, 32, 212-230. https://doi.org/10.37084/REMATEC.1980-3141.2019.n32.p212-230.id213

Sánchez, I. C., \& Prieto G, J. L. (2018). Procesos de objetivación alrededor de las ideas geométricas en la elaboración de un simulador con GeoGebra. PNA, 14(1), 230-251.

Sánchez, I. V., Prieto G., J. L., Gutiérrez, R. E., \& Díaz-Urdaneta, S. (2020). Sobre os processos de objetivação de saberes geométricos. Análise de uma experiência de elaboração de simuladores com o GeoGebra. Educación Matemática, 32(1), 99-131. https://doi.org/10.24844/EM3201.05

Sánchez, I. C., \& Sánchez, I. V. (2020). Elaboración de un simulador con GeoGebra para la enseñanza de la física. El caso de la ley de coulomb. REAMEC - Rede Amazônica de Educação Em Ciências $e$ Matemática, $\quad 8(2), \quad 40-56$. https://doi.org/10.26571/reamec.v8i2.9557

Spinoza, B. (1989). Ethics including the improvement of the understanding. (R. Elwes, Trans.). Prometheus.

Rubio, L., Prieto G., J. L., \& Ortiz, J. (2016). La matemática en la simulación con GeoGebra. Una experiencia con el movimiento en caída libre. International Journal of Educational Research and Innovation, 2, 90-111.

Vergel, R. (2014). Formas de pensamiento algebraico temprano en alumnos de cuarto y quinto grados de educación básica primaria (9-10 años) [Tesis doctoral, Universidad Distrital Francisco José de Caldas].

Autores

Ivonne Coromoto Sánchez Sánchez

Maestría en Educación en Ciencias y Matemáticas por la Universidad Federal do Pará (Brasil).

Licenciada en Educación mención Matemática y Física por la Universidad del Zulia (Venezuela). Coordinadora Administrativa de la Asociación Aprender en Red (Venezuela).

Temas de investigación: Formación de profesores; Aprendizaje matemático; Uso de tecnologías digitales en la enseñanza de la geometría. E-mail: ivonne.s.1812@gmail.com 


\section{João Cláudio Brandemberg Quaresma}

Doctor en Educación por la Universidad Federal de Rio Grande del Norte (2009). Profesor III de la Universidad Federal de Pará (UFPA) e investigador del Programa de Pós- Graduación en

Educación en Ciencias y Matemáticas del Instituto de Educación Matemática y Científica de la (UFPA). Temas de investigación: Historia de la Matemática, Enseñanza del algebra. E-mail: brand@ufpa.br

Luis Andrés Castillo Bracho Maestría en Educación en Ciencias y Matemáticas por la Universidad Federal do Pará (Brasil). Licenciado en Educación mención Matemática y Física por la Universidad del Zulia (Venezuela). Coordinador de Tecnologías Digitales y Soporte de la Asociación Aprender en Red (Venezuela). Temas de investigación: Formación de profesores; Aprendizaje matemático; Uso de tecnologías digitales en la enseñanza de la geometría. E-mail: luiscastleb@gmail.com 